\title{
Characterization of Nanoclay / Polymer Systems: A Combination of TEM, XRD and Image Analysis
}

\author{
Olivier Guise ${ }^{*}$, Al Berzinis ${ }^{*}$, Radha Kamalakaran ${ }^{* *}$ and Sushmita Franklin ${ }^{* *}$ \\ ${ }^{*}$ SABIC Innovative Plastics, 1 Noryl Avenue, Selkirk, NY 12158 - USA \\ ** GE India Technology Center, SABIC Innovative Plastics Program, Bangalore, India.
}

Nanoclays are a special class of naturally occurring aluminosilicates, which can potentially yield reinforcing plates of very high aspect ratio. Achieving complete separation (exfoliation) of the clay into these individual plates is a challenge, especially in thermoplastic resins, which are relatively non-polar. The objective is to move across the continuum from a non-exfoliated to a fully exfoliated system. While exfoliation in nanoclay polymer systems is known to directly impact properties, obtaining a quantitative measure of this is challenging. The samples are typically analyzed by transmission electron microscopy (TEM) \& X-ray diffraction (XRD), the limitations of which both can lead to misinterpretation. We therefore developed a rigorous plan to sample the materials, image them by TEM and analyze them by $x$-ray scattering, then process and analyze the images and $\mathrm{x}$-ray data. TEM is used to access the size distribution of the platelets - diameter and thickness, while XRD provides access to the inter-layer stacking distance between clay plates. Both methods are required for reliable characterization. We worked on proof-of-concept PPE/HIPS/PS blends and compared composites prepared from untreated and organo-treated nanoclays to those based on conventional fillers such as glass fiber.

We wanted to answer some basic morphology questions, as the morphology is key to interpreting property trends in phase-separated blends:

- Uniformity of clay dispersion and extent of exfoliation.

- Mean diameter and size distribution of clay plates.

- Inter-layer spacing between silicate layers.

- Any interactions between HIPS particles and nano-clays.

The results from TEM image analysis show that the distribution of the platelet diameter is lognormal for partially exfoliated organoclay in PPE/HIPS/PS. Such distribution is common in grinding processes and natural mineral samples and had been reported in the literature for the same nanoclay dispersed in water using Atomic Force Microscopy (AFM). The diameter distribution for organo-clay remains lognormal throughout a loading study from $2.5-10 \%$ by weight, and the same behavior is observed for the thickness (stack height) distribution.

The data were subsequently plugged into a modified Halpin-Tsai model to predict the system's entitlement. The Halpin-Tsai model was originally developed to predict the elastic properties of composite materials based on the geometry and orientation of the fillers and the elastic properties of the filler and the matrix, and has been modified to account for the observed nanoclay morphology of stacked plates intercalated with resin. The modified H-T model was used to estimate properties of potential filled polymer composites based on nanoclays. 

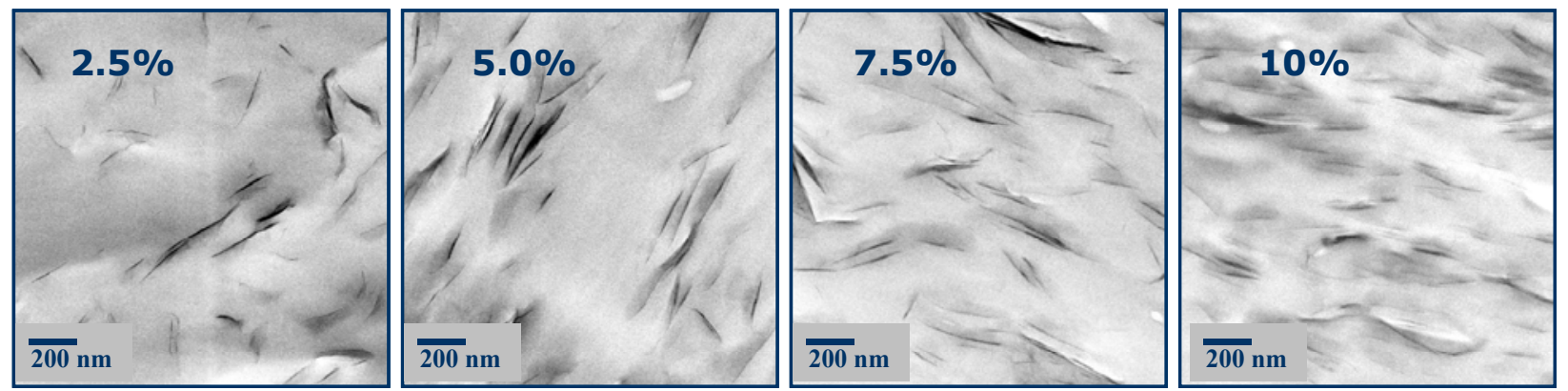

Figure 1: TEM images of organoclay at loadings of $2.5-10 \%$ by weight
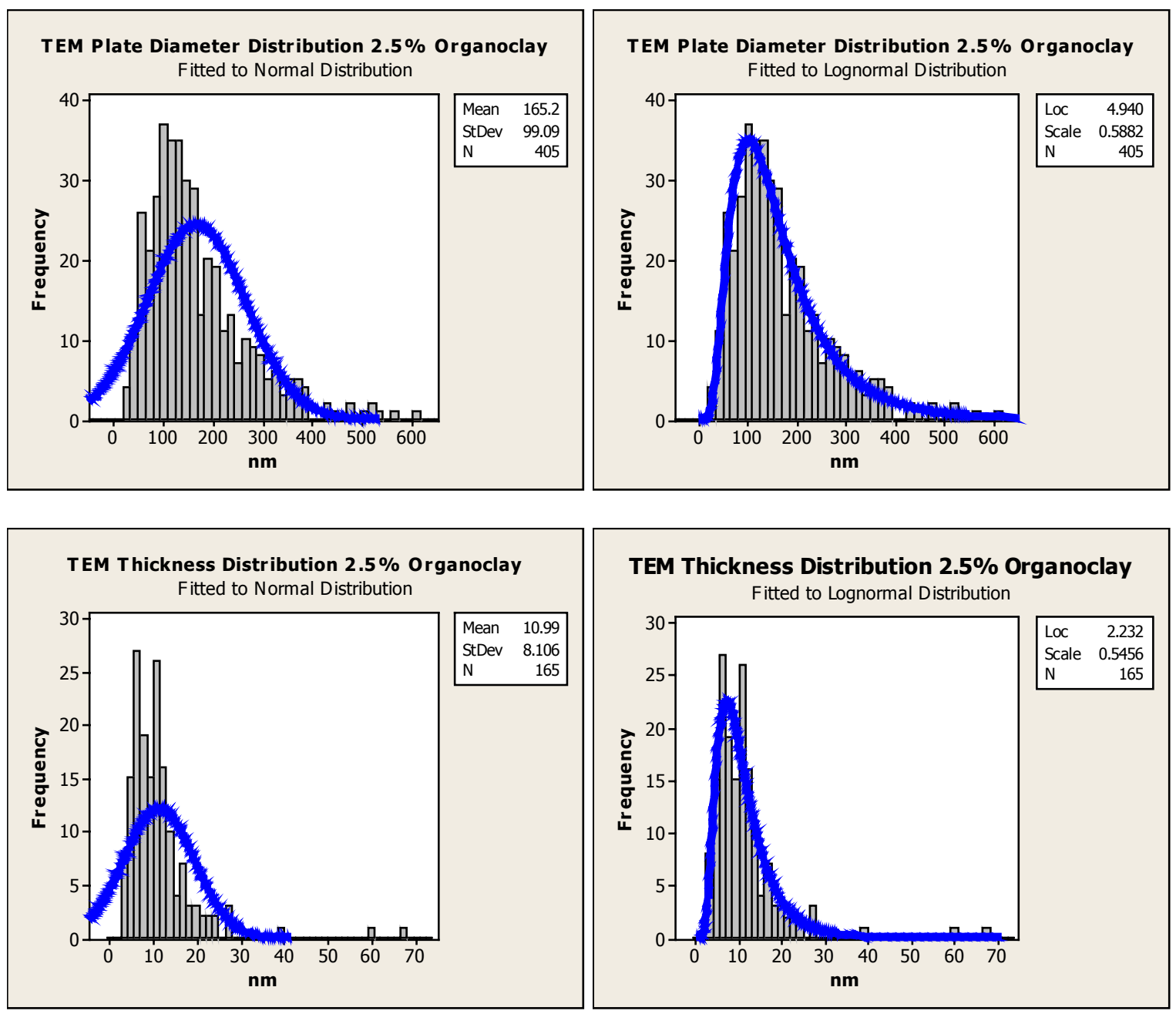

Figure 2: Platelet diameter and thickness distributions obtained from TEM image analysis of samples containing organoclay at $2.5 \%$ by weight Top Row: The platelet diameters follow a lognormal distribution. Bottom Row: The same behavior is observed for the thickness (stack height) distribution 